\title{
THE GRAVITON PROPAGATOR IN HOMOGENEOUS AND ISOTROPIC SPACETIMES
}

\author{
Bruce ALLEN \\ Department of Physics and Astronomy, Tufts University, Medford, MA 02155, USA
}

Received 28 August 1986

(Revised 19 December 1986)

\begin{abstract}
We find the propagator for gravitons in spacetimes with homogeneous and isotropic flat $(k=0)$ spatial sections. The method also applies to the $k=+1$ and $k=-1$ cases. The vacuum state is assumed to be homogeneous and isotropic in the same way as the spacetime. In the four most interesting physical cases, which are de Sitter space, the radiation and dust dominated models, and flat space, we obtain the graviton propagator in closed form for the adiabatic vacuum state.
\end{abstract}

\section{Introduction}

Spacetimes with homogeneous and isotropic spatial sections appear to be excellent models of large-scale cosmology [1,2]. To investigate certain outstanding questions, i.e., the creation of gravitons during cosmological expansion, or the behavior of particle detectors, it is useful to have closed form expressions for the propagator functions. (These are also known as Green functions or two-point correlation functions [3].) If $h^{a b}(x)$ denotes the metric perturbation at $x$, and $h^{a^{\prime} b^{\prime}}\left(x^{\prime}\right)$ the perturbation at $x^{\prime}$, then the propagators are quantum averages such as $\left\langle 0\left|h^{a b} h^{a^{\prime} b^{\prime}}\right| 0\right\rangle$. In this paper, we will find

$$
G^{a b a^{\prime} b^{\prime}}\left(x, x^{\prime}\right)=\left\langle 0\left|h^{a b}(x) h^{a^{\prime} b^{\prime}}\left(x^{\prime}\right)+h^{a^{\prime} b^{\prime}}\left(x^{\prime}\right) h^{a b}(x)\right| 0\right\rangle,
$$

which is called the symmetric function.

In order to define the propagator it is necessary to impose gauge conditions upon the metric perturbations. We require that eight conditions be satisfied [4]:

$$
\begin{array}{llrl} 
& \text { traceless: } & h^{a}{ }_{a} & =0, \\
& \text { transverse: } & \nabla_{a} h^{a b} & =0, \\
& \text { synchronous: } & t_{a} h^{a b} & =0 .
\end{array}
$$


Here $t^{a}$ is a vector orthogonal to the homogeneous and isotropic spacelike surfaces, and all tensor operations such as trace and $\nabla_{a}$ are carried out with respect to the unperturbed metric. In the case where the state $|0\rangle$ of the gravitational field is also homogeneous and isotropic, we will obtain a simple closed form for $G^{a b a^{\prime} b^{\prime}}$. The key observation is that neither the state $|0\rangle$ nor the gauge conditions (1.2) have singled out a preferred direction in the spatial sections. Furthermore, because of the gauge condition (1.2c), the propagator bitensor has only "spatial" components. Thus, if the metric is written as

$$
\mathrm{d} s^{2}=-\mathrm{d} \tau^{2}+a^{2}(\tau) \mathrm{d} \Sigma^{2},
$$

then the propagator $G^{a b a^{\prime} b^{\prime}}\left(\tau, \sigma ; \tau^{\prime}, \sigma^{\prime}\right)$ is a bitensor in $\Sigma$ which is invariant under all orientation preserving isometries that map the maximally symmetric space $\Sigma$ to itself ${ }^{\star}$.

If we further assume that the +2 and -2 helicity states of the graviton are identically occupied, then there is no preferred (right- or left-handed) orientation on the spatial sections. It then follows from the work of Allen and Jacobson [5] that $G^{a b a^{\prime} b^{\prime}}$ is a maximally symmetric bitensor for $\tau$ and $\tau^{\prime}$ fixed. For fixed $\tau$ and $\tau^{\prime}$, there are ten possible bitensors, which are

$$
\begin{array}{lll}
f_{1}(r) n^{a} n^{b} n^{a^{\prime}} n^{b^{\prime}}, & f_{5}(r) n^{a} n^{a^{\prime}} q^{b b^{\prime}}, & f_{9}(r) q^{a a^{\prime}} q^{b b^{\prime}}, \\
f_{2}(r) n^{a} n^{b} q^{a^{\prime} b^{\prime}}, & f_{6}(r) n^{b} n^{b^{\prime}} q^{a a^{\prime}}, & f_{10}(r) q^{a b^{\prime}} q^{a^{\prime} b} . \\
f_{3}(r) q^{a b} n^{a^{\prime}} n^{b^{\prime}}, & f_{7}(r) n^{a} n^{b^{\prime}} q^{a^{\prime} b}, \\
f_{4}(r) q^{a b} q^{a^{\prime} b^{\prime}}, & f_{8}(r) n^{b} n^{a^{\prime}} q^{a b^{\prime}},
\end{array}
$$

from these ten bitensors, one can form five which have the correct index symmetries $a \leftrightarrow b, a^{\prime} \leftrightarrow b^{\prime}$, and $(a b) \leftrightarrow\left(a^{\prime} b^{\prime}\right)$. Of those five bitensors, only three are traceless on the indices $a$ and $b$. Thus for arbitrary $\tau$ and $\tau^{\prime}$, the propagator must be of the form

$$
\begin{aligned}
G^{a b a^{\prime} b^{\prime}}\left(\tau, \sigma ; \tau^{\prime}, \sigma^{\prime}\right)= & P_{1}\left(\tau, \tau^{\prime}, r\right)\left(n^{a} n^{b}-\frac{1}{3} q^{a b}\right)\left(n^{a^{\prime}} n^{b^{\prime}}-\frac{1}{3} q^{a^{\prime} b^{\prime}}\right) \\
& +P_{2}\left(\tau, \tau^{\prime}, r\right)\left(q^{a a^{\prime}} q^{b b^{\prime}}+q^{a b^{\prime}} q^{b a^{\prime}}-\frac{2}{3} q^{a b} q^{a^{\prime} b^{\prime}}\right) \\
& +P_{3}\left(\tau, \tau^{\prime}, r\right)\left(4 n^{a} n^{b} n^{a^{\prime}} n^{b^{\prime}}+q^{a a^{\prime}} n^{b} n^{b^{\prime}}+q^{b a^{\prime}} n^{a} n^{b^{\prime}}\right. \\
& \left.+q^{a b^{\prime}} n^{a^{\prime}} n^{b}+q^{b b^{\prime}} n^{a} n^{a^{\prime}}\right)
\end{aligned}
$$

\footnotetext{
* This is not the same as a maximally symmetric bitensor, as defined in ref. [5]. A maximally symmetric bitensor is invariant under both orientation reversing (disconnected) isometries and orientation preserving isometries.
} 
Here $r$ is the geodesic distance between the points $\sigma$ and $\sigma^{\prime}$ in the metric $\mathrm{d} \Sigma^{2}=q_{a b} \mathrm{~d} x^{a} \mathrm{~d} x^{b}, n^{a}\left(n^{a^{\prime}}\right)$ is the unit tangent vector at the point $\sigma\left(\sigma^{\prime}\right)$ to the geodesic from $\sigma$ to $\sigma^{\prime}$ in the metric $\mathrm{d} \Sigma^{2}$, and $q_{a}^{b^{\prime}}\left(x, x^{\prime}\right)$ is the parallel propagator in the metric $\mathrm{d} \Sigma^{2}$. Thus, the problem of finding the graviton propagator is reduced to the simpler one of finding the scalar functions $P_{1}, P_{2}$ and $P_{3}$.

An important notational point is the use of primed indices, as introduced in ref. [5]. An unprimed index on a bitensor field is an index in the tangent space at the point $x$; a primed index is an index in the tangent space at the point $x^{\prime}$.

The functions $P_{i}\left(\sigma, \sigma^{\prime}, r\right)$ can be found because the mode functions for gravitons are the same as those of a massless minimally coupled scalar field [4]. In this paper we will restrict ourselves to the $k=0$ case where $\mathrm{d} \Sigma^{2}$ is the metric on flat 3-space, and we will find $P_{i}$ explicitly for the flat, dust, radiation and inflationary models. The $k=+1$ and $k=-1$ cases, in which the maximally symmetric space $\Sigma$ is a sphere or hyperbolic sheet, will be treated in a separate paper.

Note: throughout this paper we set $\hbar=c=1$. However, we retain explicitly all factors of the gravitational constant $G$.

\section{Geometric bitensors in $\boldsymbol{k}=0$ Robertson-Walker spacetimes}

In this short section, we define bitensors like those that appear in the ansatz (1.4) for the graviton propagator. In the spatially flat $(k=0)$ case there are certain advantages to using a different set of objects than those defined in the introduction. The difference is entirely in normalization factors of $a(t)$ and $a\left(t^{\prime}\right)$. To avoid any confusion, we will give these objects new names.

Let the spacetime metric be that of a Robertson-Walker model with flat spatial sections. Thus,

$$
\mathrm{d} s^{2}=a^{2}(t)\left(-\mathrm{d} t^{2}+\mathrm{d} x^{2}\right)=g_{a b} \mathrm{~d} x^{a} \mathrm{~d} x^{b} .
$$

In this section we will describe the necessary tensors and bitensors both geometrically, and in cartesian coordinates $\left(t, x_{1}, x_{2}, x_{3}\right)$. Thus in coordinates

$$
g_{a b}=a^{2}(t) \operatorname{diag}(-1,1,1,1) .
$$

Now let $t^{a}$ be a unit timelike vector field orthogonal to the spatial sections. In coordinates,

$$
t^{a}=a^{-1}(t)(1,0,0,0) .
$$

The normalization of $t^{a}$ is $t^{a} t_{a}=-1$.

Now define the projection operator $P_{a b}=g_{a b}+t_{a} t_{b}$ which projects tensors into the flat spatial section, and is the metric on a spatial surface at fixed time. In coordinates, the projection operators at $x$ and $x^{\prime}$ are

$$
P_{a b}=a^{2}(t) \operatorname{diag}(0,1,1,1), \quad P_{a^{\prime} b^{\prime}}=a^{2}\left(t^{\prime}\right) \operatorname{diag}(0,1,1,1) .
$$


The propagator $G^{a b a^{\prime} b^{\prime}}\left(x, x^{\prime}\right)$ has two points $x$ and $x^{\prime}$ as arguments. We will call the coordinates of these two points

$$
\begin{gathered}
x=(t, x)=\left(t, x_{1}, x_{2}, x_{3}\right), \\
x^{\prime}=\left(t^{\prime}, x^{\prime}\right)=\left(t^{\prime}, x_{1}^{\prime}, x_{2}^{\prime}, x_{3}^{\prime}\right) .
\end{gathered}
$$

Note again the convention that primed coordinates and indices are always associated with the point $x^{\prime}$, and that unprimed coordinates and indices are always associated with the point $x$.

We next define the comoving spatial separation of $x$ and $x^{\prime}$. We call this function $r\left(x, x^{\prime}\right)$; it is the geodesic separation of the points $x$ and $x^{\prime}$ in the time-independent static metric $a^{-2}(t) P_{a b}$. Since the spatial sections are flat, in terms of our coordinates, the distance $r$ is

$$
r\left(x, x^{\prime}\right)=\sqrt{\left(x_{1}-x_{1}^{\prime}\right)^{2}+\left(x_{2}-x_{2}^{\prime}\right)^{2}+\left(x_{3}-x_{3}^{\prime}\right)^{2}} .
$$

Now consider the geodesic $\gamma$ from $x$ to $x^{\prime}$ in the four-dimensional spacetime. We can take the tangent vectors to $\gamma$ at $x$ and $x^{\prime}$, project them into the spatial sections with $P_{a b}$ and $P_{a^{\prime} b^{\prime}}$, and then normalize them to unit length, as shown in fig. 1. In this way, we obtain a vector $V^{a}\left(x, x^{\prime}\right)$ at the point $x$ and another vector $V^{a^{\prime}}\left(x, x^{\prime}\right)$ at the point $x^{\prime}$. These vectors point away from each other, and thus away from the

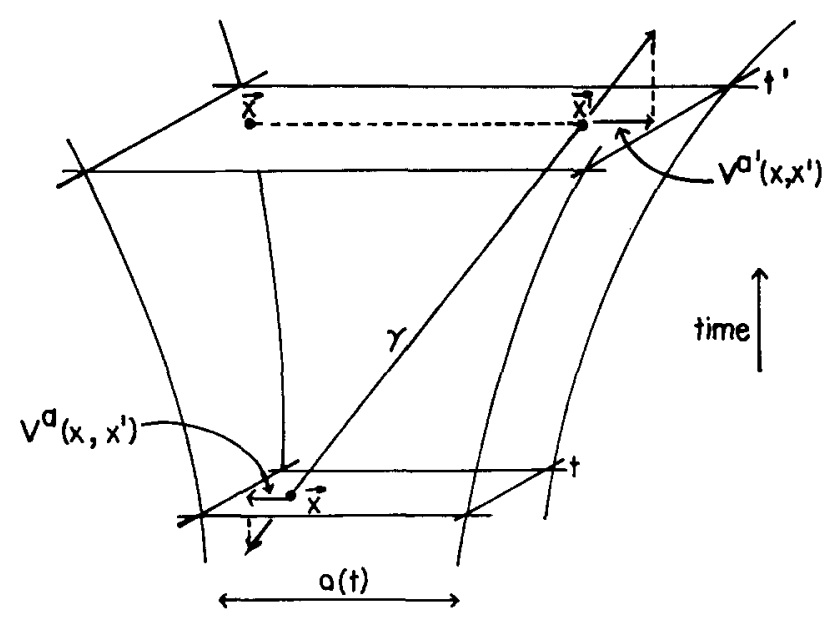

Fig. 1. Shown are two points $x=(t, x)$ and $x^{\prime}=\left(t^{\prime}, x^{\prime}\right)$ in a spatially flat Robertson-Walker model. $\gamma$ is the geodesic curve joining $x$ to $x^{\prime}$. The vector $V^{a}\left(x, x^{\prime}\right)\left(V^{a^{\prime}}\left(x, x^{\prime}\right)\right)$ is obtained by projecting the tangent vector to $\gamma$ at $x\left(x^{\prime}\right)$ onto a spacelike surface at time $t\left(t^{\prime}\right)$, and then normalizing it to unit length. Note that $V^{a^{\prime}}$ has the same direction as the geodesic in the spatial surface at time $t$ from $\left(t^{\prime}, x\right)$ to $\left(t^{\prime}, x^{\prime}\right)$. 
geodesic joining $x$ to $x^{\prime}$. In coordinates, if $\Delta x^{a}=\left(t-t^{\prime}, x-x^{\prime}\right)$ then $V^{a}=$ $a^{-1}(t) r^{-1} p_{b}^{a}(t) \Delta x^{b}$, and so

$$
\begin{aligned}
V^{a} & =a^{-1}(t) r^{-1}\left(0, x_{1}-x_{1}^{\prime}, x_{2}-x_{2}^{\prime}, x_{3}-x_{3}^{\prime}\right), \\
V^{a^{\prime}} & =a^{-1}\left(t^{\prime}\right) r^{-1}\left(0, x_{1}^{\prime}-x_{1}, x_{2}^{\prime}-x_{2}, x_{3}^{\prime}-x_{3}\right) .
\end{aligned}
$$

We can also define a parallel propagator $\delta_{c}^{c^{\prime}}$ for the time-independent spatial metric $a^{-2} P_{a b}$. In coordinates

$$
\delta_{c}^{c^{\prime}}=\operatorname{diag}(0,1,1,1)
$$

Thus, note that $V^{a^{\prime}}=-a(t) a^{-1}\left(t^{\prime}\right) \delta_{a}^{a^{\prime}} V^{a}$.

It is also convenient to define the bivector, $P_{a}^{b^{\prime}}=a(t) a^{-1}\left(t^{\prime}\right) \delta^{b^{\prime}}{ }_{b} P_{a}^{b}$

$$
P_{a}^{b^{\prime}}=a(t) a^{-1}\left(t^{\prime}\right) \operatorname{diag}(0,1,1,1) .
$$

In manipulating this bivector, note that the index $a$ transforms as a vector at $x$, and the index $b^{\prime}$ transforms as a vector at $x^{\prime}$. From these different tensors and bitensors, we can now form the graviton propagator.

Note that in the next section of this paper, the dot product of two spatial vectors $P^{a}=a^{-1}(t)(0, \boldsymbol{P})$ and $Q^{a}=a^{-1}(t)(0, \boldsymbol{Q})$ will often be denoted by $P^{a} Q_{a}=\boldsymbol{P} \cdot \boldsymbol{Q}$.

\section{Representation of $G^{a b a^{\prime} b^{\prime}}$ as a mode sum}

It has been shown by Ford and Parker that in a $k=0$ Robertson-Walker space with metric $\mathrm{d} s^{2}=a^{2}(t)\left(-\mathrm{d} t^{2}+\mathrm{d} x^{2}\right)$, the field operator for gravitational fluctuations $g_{a b} \rightarrow g_{a b}+h_{a b}$ can be represented as [4]

$$
\begin{aligned}
h^{a b}(x)= & \sum_{\boldsymbol{k}} m^{a}(\boldsymbol{k}) m^{b}(\boldsymbol{k}) F_{\mathrm{R}}(\boldsymbol{k}, x) a_{\mathrm{R}}+m^{a^{*}}(\boldsymbol{k}) m^{b^{*}}(\boldsymbol{k}) F_{\mathrm{R}}^{*}(\boldsymbol{k}, x) a_{\mathrm{R}}^{\ddagger} \\
& +\sum_{\boldsymbol{k}} m^{a^{*}}(\boldsymbol{k}) m^{b^{*}}(\boldsymbol{k}) F_{\mathrm{L}}(\boldsymbol{k}, x) a_{\mathrm{L}}+m^{a}(\boldsymbol{k}) m^{b}(\boldsymbol{k}) F_{\mathrm{L}}^{*}(\boldsymbol{k}, x) a_{\mathrm{L}}^{\ddagger}
\end{aligned}
$$

Here the operators $a_{\mathrm{R}}$ and $a_{\mathrm{R}}^{\ddagger}$ destroy and create gravitons of right-circular polarization and the operators $a_{\mathrm{L}}$ and $a_{\mathrm{L}}^{\ddagger}$ perform the same operation for left-circularly polarized gravitons ${ }^{\star}$. The sum over $\boldsymbol{k}$ denotes a sum over a three-dimen-

* This differs from Ford and Parker [4] in certain details. We use a circularly polarized basis rather than linear polarizations, we use a metric $\mathrm{d} s^{2}=a^{2}(t)\left(-\mathrm{d} t^{2}+\mathrm{d} x^{2}\right)$ rather than $\mathrm{d} t^{2}-a^{2}(t) \mathrm{d} x^{2}$, and we do not set $16 \pi G=1$. 
sional set of spatial wave vectors,

$$
\sum_{k}=V \int \mathrm{d}^{3} k
$$

In coordinates $k^{a}=a^{-1}(t)(o, k) . V$ is a constant comoving volume, which will be taken to infinity later. The complex vector $m^{a}$ is $[6,7]$

$$
m^{a}=(2)^{-1 / 2}\left[e_{1}^{a}(\boldsymbol{k})+i e_{2}^{a}(\boldsymbol{k})\right],
$$

where $e_{1}^{a}(\boldsymbol{k})$ and $e_{2}^{a}(\boldsymbol{k})$ are arbitrary vectors such that ${ }^{\star}$

(a) $e_{1}^{a}$ and $e_{2}^{a}$ are purely spatial (i.e., $P^{a}{ }_{b} e_{1}^{b}=e_{1}^{a}$ );

(b) $e_{1}^{a} e_{1}^{b} g_{a b}=e_{2}^{a} e_{2}^{b} g_{a b}=1$ and $e_{1}^{a} e_{2}^{b} g_{a b}=0$;

(c) $e_{1}^{a}$ and $e_{2}^{a}$ are orthogonal to $k$ (i.e., $e_{1}^{a} k_{a}=0$ );

(d) $e_{1}^{a}, e_{2}^{a}$ and $\boldsymbol{k}$ form a right-handed triad.

Finally the mode functions $F_{\mathrm{R}}$ and $F_{\mathrm{L}}$ are of the form

$$
F_{R}(k, x)=(16 \pi G)^{1 / 2} f_{\mathrm{R}}(k, t) \mathrm{e}^{i k \cdot x} /\left(4 \pi^{3 / 2} k^{1 / 2} V^{1 / 2}\right)
$$

and similarly for $F_{\mathrm{L}}$, where $F_{\mathrm{R}}$ satisfies the massless minimally coupled wave equation [4], and $G$ is the gravitational constant (see first footnote in this section),

$$
\square F_{\mathrm{R}}=\square F_{\mathrm{L}}=0 \text {. }
$$

Note also that $k_{a} x^{a}=k \cdot x$. The wave equation $\square F=0$ implies that the time functions $f_{\mathrm{R}}$ and $f_{\mathrm{L}}$ obey

$$
\ddot{f}+\frac{2 \dot{a}}{a} \dot{f}+k^{2} f=0,
$$

where $k=\left(k^{a} k_{a}\right)^{1 / 2}=(k \cdot k)^{1 / 2}$ and $\cdot=\mathrm{d} / \mathrm{d} t$. The time functions $f(k, t)$ are normalized by $\left(f^{*} \dot{f}-f \dot{f}^{*}\right)=-2 i k a^{-2}(t)$.

Now the symmetric two-point function in the vacuum state defined by $a_{\mathrm{L}}|0\rangle=$ $a_{\mathrm{R}}|0\rangle=0$ is given by ${ }^{\star \star}$

$$
\begin{aligned}
G^{a b a^{\prime} b^{\prime}}=\sum_{k}\{ & m^{a} m^{b} m^{a^{\prime *}} m^{b^{* *}}\left[F_{\mathrm{R}}(x) F_{\mathrm{R}}^{*}\left(x^{\prime}\right)+F_{\mathrm{L}}^{*}(x) F_{\mathrm{L}}\left(x^{\prime}\right)\right] \\
& \left.+m^{a^{*}} m^{b^{*}} m^{a^{\prime}} m^{b^{\prime}}\left[F_{\mathrm{R}}^{*}(x) F_{\mathrm{R}}\left(x^{\prime}\right)+F_{\mathrm{L}}(x) F_{\mathrm{L}}^{*}\left(x^{\prime}\right)\right]\right\} .
\end{aligned}
$$

Here $m^{a^{\prime}}=a(t) a^{-1}\left(t^{\prime}\right) m^{a} \delta_{a}^{a^{\prime}}$. If we assume that the vacuum states of the left and right-handed gravitons are the same, then $F_{\mathrm{L}}(x)=F_{\mathrm{R}}(x)=F(x)$ and we have

$$
G^{a b a^{\prime} b^{\prime}}=\sum_{k}\left(m^{a} m^{b} m^{a^{\prime *}} m^{b^{* *}}+m^{a^{*}} m^{b^{*}} m^{a^{\prime}} m^{b^{\prime}}\right)\left[F(x) F^{*}\left(x^{\prime}\right)+F^{*}(x) F\left(x^{\prime}\right)\right] \text {. }
$$

* This defines $e_{1}^{a}$ and $e_{2}^{a}$ up to a rotation by angle $\Omega$ about the $k$ axis. Under such a rotation $m^{a} m^{b} \rightarrow \exp (2 i \Omega) m^{a} m^{b}$ as discussed in ref. [6].

$\star$ Since $G^{a b a^{\prime} b^{\prime}}$ is proportional to $m^{a} m^{b} m^{c^{\prime *}} m^{d^{\prime *}}$, it is independent of the choice of polarisation vectors $e_{1}^{a}$ and $e_{2}^{a}$. 
If we further assume that the state $|0\rangle$ is homogeneous and isotropic, then the time functions $f(k, t)$ can only depend upon $k$ via its length $k$. This is because any dependence of the time functions upon the direction of $k$ would single out a preferred direction in the spatial section, thus violating the maximal symmetry of that three-dimensional space, and destroying the (assumed) homogeneity and isotropy of the graviton vacuum state.

As we explained in the introduction, the mode sum (3.8) now defines a maximally symmetric bitensor in the spatial section. We thus need to evaluate

$$
G^{a b a^{\prime} b^{\prime}}=\int\left(m^{a} m^{b} m^{a^{\prime *}} m^{b^{* *}}+m^{a^{*}} m^{b^{*}} m^{a^{\prime}} m^{b^{\prime}}\right) w\left(k, t, t^{\prime}\right) \cos k \cdot\left(\boldsymbol{x}-\boldsymbol{x}^{\prime}\right) \mathrm{d}^{3} k .
$$

The term which is proportional to $\sin \boldsymbol{k} \cdot\left(\boldsymbol{x}-\boldsymbol{x}^{\prime}\right)$ has been dropped from (3.9) since it vanishes after integration over all $k$. Note also that $k \cdot\left(x-x^{\prime}\right)=r k_{a} V^{a}$. The function $w\left(k, t, t^{\prime}\right)$ is symmetric in $\left(t, t^{\prime}\right)$ and is

$$
w\left(k, t, t^{\prime}\right)=G\left(\pi^{2} k\right)^{-1}\left[f(k, t) f^{*}\left(k, t^{\prime}\right)+f^{*}(k, t) f\left(k, t^{\prime}\right)\right] .
$$

Because the propagator (3.9) is a maximally symmetric bitensor in the spatial sections, it must be of the form

$$
G^{a b a^{\prime} b^{\prime}}=f_{1}\left(t, t^{\prime}, r\right) \theta_{1}^{a b a^{\prime} b^{\prime}}+f_{2}\left(t, t^{\prime}, r\right) \theta_{2}^{a b a^{\prime} b^{\prime}}+f_{3}\left(t, t^{\prime}, r\right) \theta_{3}^{a b a^{\prime} b^{\prime}} \text {. }
$$

This is the form that was derived in (1.4) for the specific case where the spatial sections $\Sigma$ are flat. Here the bitensors $\theta_{i}^{a b a^{\prime} b^{\prime}}\left(x, x^{\prime}\right)$ which carry the tangent space indices are:

$$
\begin{aligned}
\theta_{1}^{a b a^{\prime} b^{\prime}}= & \left(V^{a} V^{b}-\frac{1}{3} P^{a b}\right)\left(V^{a^{\prime}} V^{b^{\prime}}-\frac{1}{3} P^{a^{\prime} b^{\prime}}\right) \\
\theta_{2}^{a b a^{\prime} b^{\prime}}= & P^{a a^{\prime}} P^{b b^{\prime}}+P^{b a^{\prime}} P^{a b^{\prime}}-\frac{2}{3} P^{a b} P^{a^{\prime} b^{\prime}} \\
\theta_{3}^{a b a^{\prime} b^{\prime}}= & 4 V^{a} V^{b} V^{a^{\prime}} V^{b^{\prime}}+P^{a a^{\prime}} V^{b} V^{b^{\prime}}+P^{b a^{\prime}} V^{a} V^{b^{\prime}}+P^{a b^{\prime}} V^{b} V^{a^{\prime}} \\
& +P^{b b^{\prime}} V^{a} V^{a^{\prime}} .
\end{aligned}
$$

Then denoting $\theta^{a b a^{\prime} b^{\prime}} G_{a b a^{\prime} b^{\prime}}$ by $\theta \cdot G$ and inverting (3.11) one finds ${ }^{\star}$

$$
\left[\begin{array}{l}
f_{1} \\
f_{2} \\
f_{3}
\end{array}\right]=\frac{1}{8}\left[\begin{array}{rrr}
27 & -3 & -3 \\
-3 & 1 & 1 \\
-3 & 1 & 2
\end{array}\right]\left[\begin{array}{l}
\theta_{1} \cdot G \\
\theta_{2} \cdot G \\
\theta_{3} \cdot G
\end{array}\right] .
$$

To evaluate the quantities $\theta_{i}^{a b a^{\prime} b^{\prime}}\left(m_{a} m_{b} m_{a^{\prime}}^{*} m_{b^{\prime}}^{*}+m_{a}^{*} m_{b}^{*} m_{a^{\prime}} m_{b^{\prime}}\right)$ it now helps to

* This inversion is assisted by the following contractions: $\theta_{1} \cdot \theta_{1}=\frac{4}{9}, \theta_{1} \cdot \theta_{2}=\frac{4}{3}, \theta_{1} \cdot \theta_{3}=0, \theta_{2} \cdot \theta_{2}=20$, $\theta_{2} \cdot \theta_{3}=-8, \theta_{3} \cdot \theta_{3}=8$. 
introduce a special set of $e_{1}^{a}$ and $e_{2}^{a}$. Choose $e_{1}^{a}$ to be orthogonal to both $k^{a}$ and $V^{a}$, and $e_{2}^{a}$ to be orthogonal to both $e_{1}^{a}$ and $k^{a}$ (see the discussion following (3.3) and the first footnote in this section). Then

$$
\begin{aligned}
& \theta_{1} \cdot G=\frac{1}{2} \int\left(V_{a} e_{2}^{a}\right)^{4} w\left(k, t, t^{\prime}\right) \cos \boldsymbol{k} \cdot\left(\boldsymbol{x}-\boldsymbol{x}^{\prime}\right) \mathrm{d}^{3} k, \\
& \theta_{2} \cdot G=4 \int w\left(k, t, t^{\prime}\right) \cos \boldsymbol{k} \cdot\left(\boldsymbol{x}-\boldsymbol{x}^{\prime}\right) \mathrm{d}^{3} k, \\
& \boldsymbol{\theta}_{3} \cdot G=2 \int\left[\left(V_{a} e_{2}^{a}\right)^{4}-2\left(V_{a} e_{2}^{a}\right)^{2}\right] w\left(k, t, t^{\prime}\right) \cos \boldsymbol{k} \cdot\left(\boldsymbol{x}-\boldsymbol{x}^{\prime}\right) \mathrm{d}^{3} k .
\end{aligned}
$$

Since $w\left(k, t, t^{\prime}\right)$ does not depend upon the direction of $\boldsymbol{k}$, these integrals are easily evaluated.

To do this, first note that $\boldsymbol{k} \cdot\left(\boldsymbol{x}-\boldsymbol{x}^{\prime}\right)=r k^{a} V_{a}=k r \cos \phi$ where $\phi$ is the angle between $k^{a}$ and $V^{a}$. Because of the above choice of $e_{1}^{a}$ and $e_{2}^{a}$ it then follows that $\left(V_{a} e_{2}^{a}\right)^{2}=\sin ^{2} \phi=1-\cos ^{2} \phi$. Since the measure $\mathrm{d}^{3} k=2 \pi k^{2} \mathrm{~d} k \sin \phi \mathrm{d} \phi$ for $k \in[0, \infty)$ and $\phi \in[0, \pi]$, we obtain, for example

$$
\int\left(V_{a} e_{2}^{a}\right)^{2} w(k) \cos k \cdot\left(x-x^{\prime}\right) \mathrm{d}^{3} k=2 \pi \int_{0}^{\infty} w(k) k^{2}\left\{\int_{0}^{\pi} \sin ^{3} \phi \mathrm{e}^{i k r \cos \phi} \mathrm{d} \phi\right\} \mathrm{d} k
$$

The integral over $\phi$ may be evaluated by letting $u=\cos \phi$ and integrating over $u$. The integral over $\phi$ (in curly brackets above) then becomes, for example,

$$
4\left(\frac{\sin k r}{k^{3} r^{3}}-\frac{\cos k r}{k^{2} r^{2}}\right)
$$

In this way we obtain for the different integrals in (3.14)

$$
\begin{gathered}
\int w(k) \mathrm{e}^{i k \cdot x} \mathrm{~d}^{3} k=\frac{4 \pi}{r} \int_{0}^{\infty} w(k)(\sin k r) k \mathrm{~d} k, \\
\int\left(V_{a} e_{2}^{a}\right)^{2} w(k) \mathrm{e}^{i k \cdot x} \mathrm{~d}^{3} k=\frac{4 \pi}{r} \int_{0}^{\infty} w(k)\left[\frac{2 \sin k r}{k^{2} r^{2}}-\frac{2 \cos k r}{k r}\right] k \mathrm{~d} k, \\
\int\left(V_{a} e_{2}^{a}\right)^{4} w(k) \mathrm{e}^{i k \cdot x} \mathrm{~d}^{3} k=\frac{4 \pi}{r} \int_{0}^{\infty} w(k)\left[\frac{24 \sin k r}{k^{4} r^{4}}-\frac{24 \cos k r}{k^{3} r^{3}}-\frac{8 \sin k r}{k^{2} r^{2}}\right] k \mathrm{~d} k .
\end{gathered}
$$


Thus from (3.13) and (3.14) we find, for the functions $f_{1}, f_{2}$ and $f_{3}$

$$
\left[\begin{array}{l}
f_{1}\left(t, t^{\prime}, r\right) \\
f_{2}\left(t, t^{\prime}, r\right) \\
f_{3}\left(t, t^{\prime}, r\right)
\end{array}\right]=2 \pi\left[\begin{array}{rrrrr}
-3 & -9 & 45 & -6 & -45 \\
1 & -3 & 3 & 2 & -3 \\
1 & -9 & 15 & 4 & -15
\end{array}\right]\left[\begin{array}{l}
s_{1}\left(t, t^{\prime}, r\right) \\
s_{3}\left(t, t^{\prime}, r\right) \\
s_{5}\left(t, t^{\prime}, r\right) \\
c_{2}\left(t, t^{\prime}, r\right) \\
c_{4}\left(t, t^{\prime}, r\right)
\end{array}\right]
$$

where

$$
\begin{aligned}
& s_{n}\left(t, t^{\prime}, r\right)=\int_{0}^{\infty} \frac{\sin k r}{k^{n} r^{n}} w\left(k, t, t^{\prime}\right) k^{2} \mathrm{~d} k, \\
& c_{n}\left(t, t^{\prime}, r\right)=\int_{0}^{\infty} \frac{\cos k r}{k^{n} r^{n}} w\left(k, t, t^{\prime}\right) k^{2} \mathrm{~d} k .
\end{aligned}
$$

Thus to obtain the graviton propagator in a homogeneous and isotropic spacetime, one must carry out the following steps:

(i) Choose the time-parts of the mode functions $f(k, t)$ such that

(a) $f(k, t)$ only depends upon the length $k$ of $k$,

(b) $f(k, t)$ satisfies $\ddot{f}+2(\dot{a} / a) \dot{f}+k^{2} f=0$,

(c) $f(k, t)$ is normalized so that $f^{*} \dot{f}-f f^{*}=-2 i k a^{-2}(t)$.

(ii) Form the quantity $w\left(k, t, t^{\prime}\right)$ from $f(k, t)$ :

$$
w\left(k, t, t^{\prime}\right)=G\left(\pi^{2} k\right)^{-1}\left[f^{*}(k, t) f\left(k, t^{\prime}\right)+f(k, t) f^{*}\left(k, t^{\prime}\right)\right] .
$$

(iii) Evaluate the five one-dimensional integrals $s_{1}, s_{3}, s_{5}, c_{2}, c_{4}$ defined by (3.17).

(iv) Use (3.16) to find $f_{1}, f_{2}$ and $f_{3}$. The symmetric function $G^{a b a^{\prime} b^{\prime}}\left(x, x^{\prime}\right)$ is then given by (3.11).

The choice of a vacuum state $|0\rangle$ is implicit in step 1 above. This is because the requirement that the state $|0\rangle$ be Robertson-Walker invariant (i.e., homogeneous and isotropic) does not single out a unique state. In fact, there are an infinite number of choices of functions $f(k, t)$, and corresponding "vacuum" states $|0\rangle$, that satisfy the necessary conditions given above.

\section{Special cases: de Sitter, radiation and dust models}

In this sectior. we evaluate the graviton propagator in closed form for four special spacetimes. They are de Sitter space, the radiation dominated model, the dust dominated model, and flat space. In each of these models the vacuum state is chosen to be the infinite-order adiabatic vacuum state [3]. In the de Sitter model this vacuum state is also called the Bunch-Davies vacuum state [8]. In the radiation model, it is the same state as the Minkowski conformal vacuum state [3]. 
The scale factors $a(t)$ and mode function $f(k, t)$ are as follows:

$$
\begin{array}{ll}
\text { de Sitter space: } & a(t)=(H t)^{-1} \\
& f(k, t)=\left(a^{-1}(t)-i H k^{-1}\right) \exp (-i k t) ; \\
\text { radiation dominated: } & a(t)=H t, \\
& f(k, t)=a^{-1}(t) \exp (-i k t) ; \\
\text { dust dominated: } & a(t)=(H t)^{2}, \\
& f(k, t)=\left(a^{-1}(t)-i H k^{-1} a^{-3 / 2}(t)\right) \exp (-i k t) ; \\
& a(t)=1, \\
\text { flat space: } & f(k, t)=\exp (-i k t) .
\end{array}
$$

Here the constant $H$ determines the expansion rate: in the case of de Sitter space it is equal to the Hubble constant. Note that since the metric is $\mathrm{d} s^{2}=a^{2}(t)\left(-\mathrm{d} t^{2}+\right.$ $\left.\mathrm{d} x^{2}\right)$, the coordinate $t$ is "conformal" time and not the more physical "comoving" time.

For these four spacetimes, the $t_{\text {unction }} w\left(k, t, t^{\prime}\right)$ defined in (3.10) takes the simple form

$$
\begin{aligned}
w\left(k, t, t^{\prime}\right)= & 2 G \pi^{-2} A\left(t, t^{\prime}\right)\left[k^{-3} \cos k\left(t-t^{\prime}\right)+k^{-2}\left(t-t^{\prime}\right) \sin k\left(t-t^{\prime}\right)\right] \\
& +2 G \pi^{-2} B\left(t, t^{\prime}\right) k^{-1} \cos k\left(t-t^{\prime}\right) .
\end{aligned}
$$

The functions $A\left(t, t^{\prime}\right)$ and $B\left(t, t^{\prime}\right)$ can be found from (4.1)-(4.4). These are as follows:

de Sitter space: $\quad A\left(t, t^{\prime}\right)=H^{2}$,

$$
B\left(t, t^{\prime}\right)=a^{-1}(t) a^{-1}\left(t^{\prime}\right) ;
$$

radiation:

$$
A\left(t, t^{\prime}\right)=0 \text {, }
$$

$$
B\left(t, t^{\prime}\right)=a^{-1}(t) a^{-1}\left(t^{\prime}\right) ;
$$

dust:

$$
\begin{aligned}
& A\left(t, t^{\prime}\right)=H^{2} a^{-3 / 2}(t) a^{-3 / 2}\left(t^{\prime}\right), \\
& B\left(t, t^{\prime}\right)=a^{-1}(t) a^{-1}\left(t^{\prime}\right) ;
\end{aligned}
$$

flat:

$$
\begin{aligned}
& A\left(t, t^{\prime}\right)=0, \\
& B\left(t, t^{\prime}\right)=1 .
\end{aligned}
$$


Now, in order to find the propagator, we need to find the functions $f_{1}, f_{2}$ and $f_{3}$ that appear in (3.11). They are given by (3.16) in terms of integrals that we can now evaluate.

In particular, we need to know the integral

$$
\begin{aligned}
\int_{m}^{\infty} k^{-6} & \sin k r \cos k t \mathrm{~d} k \\
= & \frac{1}{4} r m^{-4}-\frac{1}{12} r\left(3 t^{2}+r^{2}\right) m^{-2}-\frac{1}{480} t\left(t^{4}+10 t^{2} r^{2}+5 r^{4}\right) \log \left(\frac{r+t}{r-t}\right)^{2} \\
& \quad-\frac{1}{480} r\left(r^{4}+10 r^{2} t^{2}+5 t^{4}\right)\left[\log \left(m^{4}\left(t^{2}-r^{2}\right)^{2}\right)+4 \gamma-\frac{137}{15}\right]+\mathrm{O}\left(m^{2}\right) .
\end{aligned}
$$

Here $m$ is an infra-red cutoff which we would like to take to zero, and $\gamma$ is Euler's constant $\gamma=0.5772 \ldots$. By taking repeated derivatives of this integral with respect to $r$ and $t$ we can find all of the integrals necessary to evaluate $f_{1}, f_{2}$ and $f_{3}$ when $w\left(k, t, t^{\prime}\right)$ has the form (4.5).

Evaluating the integrals $s_{1}, s_{3}, s_{5}, c_{2}$ and $c_{4}$ we thus obtain,

$$
\begin{array}{r}
{\left[\begin{array}{l}
f_{1} \\
f_{2} \\
f_{3}
\end{array}\right]=2 G \pi^{-1} A\left(t, t^{\prime}\right)\left[\begin{array}{r}
\frac{3}{4} V^{2}\left(V^{2}-3\right) \psi_{2}-\frac{1}{5}\left(15 V^{4}-40 V^{2}-12\right) \\
-\frac{1}{5} \psi_{1}+\frac{1}{20} V^{2}\left(V^{2}+5\right) \psi_{2}-\frac{1}{75}\left(15 V^{4}+80 V^{2}-32\right) \\
\frac{1}{4} V^{2}\left(V^{2}+1\right) \psi_{2}-\frac{1}{15}\left(15 V^{4}+20 V^{2}+8\right)
\end{array}\right]} \\
+2 G \pi^{-1} B\left(t, t^{\prime}\right) r^{-2}\left[\begin{array}{c}
\frac{3}{4}\left(5 V^{2}-9\right) \psi_{2}+\left(1-V^{2}\right)^{-1}\left(15 V^{4}-37 V^{2}+16\right) \\
\frac{1}{4}\left(V^{2}+3\right) \psi_{2}+\frac{1}{3}\left(1-V^{2}\right)^{-1}\left(3 V^{4}+7 V^{2}-4\right) \\
\frac{1}{4}\left(5 V^{2}+3\right) \psi_{2}+\frac{1}{3}\left(1-V^{2}\right)^{-1}\left(15 V^{4}-V^{2}-8\right)
\end{array}\right],
\end{array}
$$

where the variable $V$ and functions $\psi_{1}$ and $\psi_{2}$ are defined as follows:

$$
\begin{aligned}
V & =\left(t-t^{\prime}\right) / r \\
\psi_{1} & =\log \left(m^{4} r^{4}\left(1-V^{2}\right)^{2}\right)+4 \gamma, \\
\psi_{2} & =V \log \left(\frac{1+V}{1-V}\right)^{2}
\end{aligned}
$$

Notice that the infra-red cutoff $\mathrm{m}^{2}$ appears only in the function $\psi_{1}$ and thus that it has disappeared from the propagator except for appearing in a single term of $f_{2}$. What is the physical significance of this term? 
It is well-known that the minimally coupled massless scalar field suffers from an infra-red divergence in the de Sitter and dust models, where $A\left(t, t^{\prime}\right)$ is nonzero $[4,9-12]$. It is easy to see that the following trace of the graviton propagator,

$$
P_{b}{ }^{b^{\prime}} P_{a} a^{\prime} G^{a b}{ }_{a^{\prime} b^{\prime}}=\frac{2}{3} f_{1}+10 f_{2}-4 f_{3}=(16 \pi G) 2 G_{\text {scalar }}^{(1)}
$$

is (apart from a factor of $16 \pi \mathrm{G}$ ) exactly twice the massless minimally coupled scalar propagator $G_{\text {scalar }}^{(1)}$. Thus, the infra-red divergence of the scalar field appears in the graviton propagator also. This means that for the choice of gauge which we have made (1.21), the graviton propagator has an infra-red divergence in de Sitter space and in the dust model. That is to say, in the limit $m \rightarrow 0$, the graviton propagator (in our gauge) is infinite in these models.

It has been shown that in de Sitter space there are certain "bad" choices of gauge for which the graviton propagator contains infinite terms; however, for these choices of gauge the infinite terms do not contribute to any physical process [13]. Indeed, there exist other choices of gauge, for which the graviton propagator is completely finite. It follows that for a "bad" choice of gauge, an infinite term in the graviton propagator cannot contribute to the scattering amplitude. This is because the scattering amplitude is a physical, and thus gauge-independent, quantity. In our present case, we can write the graviton propagator in the form

$$
G^{a b a^{\prime} b^{\prime}}=G_{\text {finite }}^{a b a^{\prime} b^{\prime}}-\frac{4}{5} \pi^{-1} G A\left(t, t^{\prime}\right) \log \left(m^{2}\right) \theta_{2}^{a b a^{\prime} b^{\prime}},
$$

where $\theta_{2}^{a b a^{\prime} b^{\prime}}$ is the tensor defined in (3.12) which is formed entirely from the projected metric tensor, and $G_{\text {finite }}^{a b a^{\prime} b^{\prime}}$ is a finite term, independent of $m$.

In de Sitter space, the second term on the right-hand side of (4.14) does not contribute to tree-level scattering amplitudes. Thus it is a gauge artifact - this term contributes nothing, for any value of the cutoff parameter $\mathrm{m}^{2}$. This is because the tree-level scattering amplitude is given by a double integral of the form [13]

$$
S=\iint T_{a b}(x) G^{a b a^{\prime} b^{\prime}}\left(x, x^{\prime}\right) T_{a^{\prime} b^{\prime}}\left(x^{\prime}\right) \sqrt{g} \mathrm{~d}^{4} x \sqrt{g^{\prime}} \mathrm{d}^{4} x^{\prime} .
$$

The stress-energy tensor $T_{a b}$, for any interaction, is conserved $\nabla^{a} T_{a b}=0$ and symmetric $T_{a b}=T_{b a}$. In de Sitter space $A\left(t, t^{\prime}\right)=$ constant and $\theta_{2}^{a b a^{\prime} b^{\prime}}=\nabla^{(a} Q^{b) a^{\prime} b^{\prime}}$ where the bitensor $Q^{b a^{\prime} b^{\prime}}$ is

$$
Q^{b a^{\prime} b^{\prime}}=H^{-1}\left(\frac{2}{3} t^{b} P^{a^{\prime} b^{\prime}}-2(r / t) V^{a^{\prime}} P^{b b^{\prime}}\right) .
$$

* This is easily verified with the formulae (true for any scale-factor $a(t)$ )

$$
\begin{aligned}
\nabla_{a} t_{b} & =\dot{a}(t) a^{-2}(t) P_{a b}, \\
\nabla_{a} P^{b b^{\prime}} & =\dot{a}(t) a^{-2}(t) t^{b} P_{a}^{b^{\prime}}, \\
\nabla_{a}\left(r V^{b^{\prime}}\right) & =-a^{-1}(t) P_{a}^{b^{\prime}} .
\end{aligned}
$$

Here 'denotes $\mathrm{d} / \mathrm{d} t$. 
Thus one can integrate by parts in (4.15) to show that the second term in (4.14) does not contribute to the scattering amplitude (4.15) - it is a gauge artifact!

It is interesting to examine the behavior of the propagator for large spacelike separations, i.e., as $r \rightarrow \infty$ with $t$ and $t^{\prime}$ held fixed. We then find that for large $r$,

$$
\left[\begin{array}{l}
f_{1} \\
f_{2} \\
f_{3}
\end{array}\right] \rightarrow \frac{2}{5} \pi^{-1} G A\left(t, t^{\prime}\right)\left[\begin{array}{c}
12 \\
\frac{32}{15}-2 \log \left(m^{2} r^{2}\right)-4 \gamma \\
-\frac{8}{3}
\end{array}\right]+\mathrm{O}\left(1 / r^{2}\right) .
$$

It is not clear what the significance of this is. In particular, the fact that $f_{2}$ grows logarithmically at large $r$ may be a gauge artifact because the rate of growth depends upon $\mathrm{m}^{2}$, and as we have already argued, at least in de Sitter space, any scattering diagram is independent of $m^{2}$. Unfortunately, in de Sitter space, one can prove that $\log r^{2} \theta_{2}^{a b a^{\prime} b^{\prime}}$ is not of the form $\nabla^{(a} W^{b) a^{\prime} b^{\prime}}$ for any choice of $W^{b a^{\prime} b^{\prime}}$. Thus one can not show that the logarithmically growing part of the propagator makes no contribution to tree-level scattering amplitudes. Equally curious is the fact that the remaining part of the propagator approaches a constant value at large $r$. If one thinks of the propagator as giving the response of the metric to a small perturbation, then this means that instead of dying out at large separations, the small metric perturbations "freeze".

\section{Conclusion}

We have shown how to find the graviton propagator in any $k=0$ RobertsonWalker spacetime. Our method requires only that the state be invariant under the six-parameter isometry group of the spacelike surfaces. We have further assumed that there is an identical occupation of right-handed and left-handed graviton modes. This amounts to demanding that the state in question be invariant under parity transformations, which have the effect of reversing the orientation of the three-surfaces. This last assumption can be removed, but there are then additional terms beyond the three which we have given, that appear in the graviton propagator. These terms have an appearance similar to (3.12) but involve in addition the orientation tensor $\varepsilon^{a b c}$ of the three-surface ${ }^{\star}$. They will be treated in a later publication.

The method which we have given can be generalized to the $k=+1$ and $k=-1$ cases. In particular, provided that the state is parity-invariant, homogeneous, and isotropic, the propagator will take the form (1.4) of a maximally symmetric bitensor in the three-space. Formulae analogous to (3.16), which give the coefficient func-

ॠ The existence of such terms is a consequence of the identity $2 m^{a} m^{b^{*}}=P^{a b}-\hat{k}^{a} \hat{k}^{b}+i \varepsilon^{a b c} \hat{k}_{c}$. Here $m^{a}$ is defined in (3.3), and $\hat{k}^{a}$ is the unit vector $k^{a} / k$. If the graviton state is not parity invariant, then the propagator contains terms such as $\left.V_{e} V^{(b} \varepsilon^{a) e(c} V^{d}\right) \delta_{c}^{a^{\prime}} \delta_{a}^{b^{\prime}}$. 
tions of the three bitensors appearing in the propagator, will also be given in a later publication.

In the $k=0$ case the graviton propagator is given in terms of three scalar functions $f_{i}\left(t, t^{\prime}, r\right)$. These functions are ordinary one-dimensional integrals of the massless scalar mode functions, which we have evaluated in closed form for the flat, dust, de Sitter and radiation models. In fact, the integrals can probably be performed in closed form for any scale factor $a$ which is a power law $a \sim t^{k}$ in conformal time $t$ [14].

This work may be of importance in regard to recent claims that de Sitter space is unstable, either intrinsically [15] or in the presence of matter fields [11,16-20]. In particular, the propagator has an infra-red divergence, and it grows logarithmically for large spacelike separations. The (infinite) infra-red divergence is a harmless gauge artifact [13], but the large-distance behavior of the propagator may well be symptomatic of some fundamental physical instability. However, since the propagator itself is not a gauge-invariant object, further investigation will be necessary to understand this point.

I would like to thank Larry Ford and Ted Jacobson for several helpful conversations. This work has been partly supported by NSF grant $83-51860$ and by a grant from the General Electric Company.

\section{References}

[1] A.K. Raychaudhuri, Theoretical cosmology (Oxford University Press, London, 1979)

[2] S.W. Hawking and G.F.R. Ellis, The large scale structure of space-time (Cambridge University Press, London, 1980)

[3] N.D. Birrell and P.C.W. Davies, Quantum fields in curved space (Cambridge University Press, London, 1982)

[4] L.H. Ford and Leonard Parker, Phys. Rev. D16 (1977) 1601

[5] B. Allen and T. Jacobson, Comm. Math. Phys. 103 (1986) 669

[6] R.P. Feynman, Lectures on gravitation 1962-63, ed. F.B. Morinigo and W.G. Wagner (California Institute of Technology, 1971)

[7] S. Weinberg, Gravitation and cosmology (Wiley, New York, 1972)

[8] Bruce Allen, Phys. Rev. D32 (1985) 3136

[9] Alexander Vilenkin and L.H. Ford, Phys. Rev. D26 (1982) 123

[10] L.H. Ford, Phys. Rev. D31 (1985) 704

[11] L.H. Ford, Phys. Rev. D31 (1985) 710

[12] L.H. Ford and Alexander Vilenkin, Phys. Rev. D33 (1986) 2833

[13] B. Allen, Phys. Rev. D34 (1986) 3670

[14] L.H. Ford and D.J. Toms, Phys. Rev. D25 (1982) 1510 (see, e.g., eq. (4.18))

[15] I. Antoniadis, J. Iliopoulos and T.N. Tomaras, Phys. Rev. Lett. 56 (1986) 1319

[16] E. Mottola, Phys. Rev. D31 (1985) 754

[17] E. Mottola, Phys. Rev. D33 (1986) 1616

[18] E. Mottola, NSF-ITP 85-35 preprint, to appear in Phys. Rev. D (1986)

[19] P. Mazur and E. Mottola, Nucl. Phys. B278 (1986) 694

[20] N. P. Myhrvold, Phys. Rev. D28 (1983) 2439 